\title{
2D refractive index field measurements in air in different pressure scenarios
}

Beermann, Rüdiger, Quentin, Lorenz, Reithmeier, Eduard, Kästner, Markus

Rüdiger Beermann, Lorenz Quentin, Eduard Reithmeier, Markus Kästner, "2D refractive index field measurements in air in different pressure scenarios," Proc. SPIE 10819, Optical Metrology and Inspection for Industrial Applications V, 108190E (2 November 2018); doi: 10.1117/12.2500315 


\title{
2D Refractive Index Field Measurements in Air in Different Pressure Scenarios
}

\author{
Rüdiger Beermann ${ }^{\mathrm{a}}$, Lorenz Quentin ${ }^{\mathrm{a}}$, Eduard Reithmeier ${ }^{\mathrm{a}}$, and Markus Kästner ${ }^{\mathrm{a}}$ \\ ${ }^{a}$ Institute of Measurement and Automatic Control, Leibniz Universität Hannover, Nienburger \\ Str. 17, 30167 Hannover, Germany
}

\begin{abstract}
The optical geometry characterization of wrought hot components can help to quantify material distortion effects during air-cooling. The component's shrinkage behavior is affected by inhomogeneous heat dissipation due to the object's complex geometry and - in case of hybrid materials - differing thermal expansion coefficients. As optical triangulation techniques rely on the rectilinear expansion of light, the hot component's heat input into the surrounding medium air influences the reachable accuracy of optical geometry measurements due to an inhomogeneous refractive index field around the hot component. In previous work, the authors identified low pressure measurements in air as a possible approach to reduce the magnitude and expansion of the inhomogeneous refractive index field above cylindrical high-temperature objects and thereby allow precise geometry acquisition. We now present experimental data of the $2 \mathrm{D}$ refractive index field above a hot cylinder in different pressure scenarios using the well-known background oriented schlieren (BOS) method in order to illustrate the decrease in refractive index variations dependent on the pressure state. For this purpose, a ceramic rod is placed in a vacuum chamber and heated up to temperatures of about $1000^{\circ} \mathrm{C}$. Using a monochromatic camera, a wavelet background and an optical flow algorithm, the developing 2D refractive index field for a low pressure scenario is compared to ambient pressure conditions. The experimental data illustrates a reduction in the convective heat flow above the hot heating rod at lower pressure values and therefore a homogenization of the density-coupled refractive index in air, validating former simulation results.
\end{abstract}

Keywords: 3D measurement, triangulation, fringe projection, refractive index field, light deflection, optical inhomogeneity

\section{INTRODUCTION}

Within the Collaborative Research Centre 1153 (CRC) Process Chain for Manufacturing Hybrid High Performance Components by Tailored Forming new techniques are being investigated to produce hybrid workpieces made form different materials. The main objective of subproject C5 located at the Institute of Measurement and Automatic Control is the research on measurement techniques to fully characterize the geometry of the wrought-hot hybrid workpieces directly after forming. An immediate condition-monitoring of the so-called Tailored Forming-components can yield advantages - such as the reduction of production costs due to an early detection of rejects or a full documentation of the shrinkage behavior to quantify material distortion effects. After the forming process, the Tailored Forming-components have temperatures above $1000{ }^{\circ} \mathrm{C}-$ therefore the inspection method needs to be optical.

To realize fast and area-wise geometry measurements, the fringe projection method has been identified as an appropriate measurement technique for the condition-monitoring of Tailored Forming-workpieces. Based on the triangulation principle, the fringe projection method relies on the rectilinear projection of light on and reflection of light from the measurement object's surface. As the air around the hot-wrought workpieces is heated up, an inhomogeneous density gradient field develops around the object, which in turn causes a 3D inhomogeneous refractive index field. Light traversing such an inhomogeneous field is bent towards optically more dense layers, leading to a violation of the triangulation principle. Depending on the lateral sensor resolution, the reachable accuracy of the optical sensor system might be reduced.

Further author information: (Send correspondence to Rüdiger Beermann)

Rüdiger Beermann: E-mail: ruediger.beermann@imr.uni-hannover.de, Telephone: +49511 7625816

Optical Metrology and Inspection for Industrial Applications V, edited by Sen Han,

Toru Yoshizawa, Song Zhang, Proc. of SPIE Vol. 10819, 108190E · @ 2018 SPIE

CCC code: $0277-786 \mathrm{X} / 18 / \$ 18 \cdot$ doi: $10.1117 / 12.2500315$

Proc. of SPIE Vol. 10819 108190E-1 


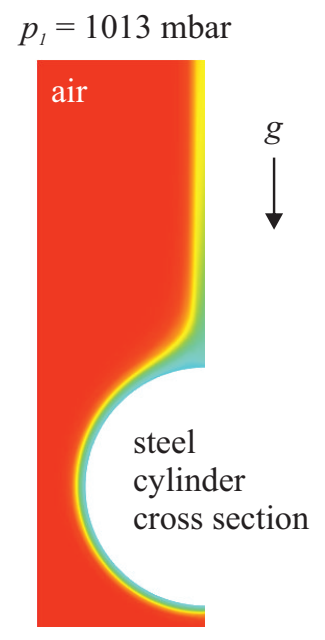

$$
\begin{aligned}
& \rho_{\text {max }}=1.18 \mathrm{~kg} / \mathrm{m}^{3} \\
& \rho_{\text {min }}=0.517 \mathrm{~kg} / \mathrm{m}^{3}
\end{aligned}
$$

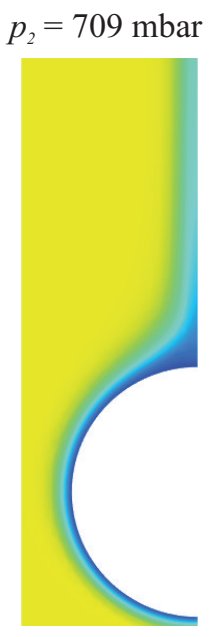

$\rho_{\text {max }}=0.828 \mathrm{~kg} / \mathrm{m}^{3}$
$\rho_{\text {min }}=0.361 \mathrm{~kg} / \mathrm{m}^{3}$ $p_{3}=355 \mathrm{mbar} \quad \rho\left(\mathrm{kg} / \mathrm{m}^{3}\right)$

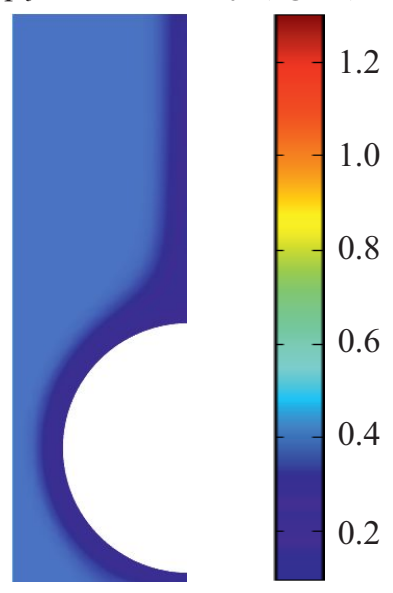

$\rho_{\max }=0.413 \mathrm{~kg} / \mathrm{m}^{3}$

$\rho_{\text {min }}=0.18 \mathrm{~kg} / \mathrm{m}^{3}$

Figure 1: 2D density simulations around the cross section of a hot steel cylinder with a diameter of $10 \mathrm{~cm}$ for different pressure scenarios. Only the left side of the cross section is modeled, as a symmetric field is building up. The start temperature of the cylinder is $420^{\circ} \mathrm{C}$, the start temperature of the surrounding humid air is $25^{\circ} \mathrm{C}$. The density maps are gained after a simulation time of $6 \mathrm{~min}$. Clearly visible is the convective density flow above the cylinder.

\section{MOTIVATION}

In previous work, the authors identified low pressure measurements in air as a possible approach to reduce the variation of inhomogeneous refractive index fields above cylindrical high-temperature objects to allow precise geometry acquisition. ${ }^{1}$ The idea of this approach is simple: As the heat induced 3D density gradient field in air causes an undesired optical inhomogeneity, a reduction of air molecules might positively affect the variation of the field, and thereby also affect the expansion and magnitude of the inhomogeneous refractive index field.

2D simulations of the density field around the cross section of a hot steel cylinder for different pressure scenarios after a simulation time of 6 min are displayed in figure 1 . To save calculation costs, symmetry effects are exploited and only the left side of the cross section is modeled. The steel cylinder has a start temperature of $420^{\circ} \mathrm{C}$ and it's diameter is $10 \mathrm{~cm}$. The surrounding medium is defined as humid air with a relative humidity of $55 \%$ and a start temperature of $25^{\circ} \mathrm{C}$. Furthermore, the fluid flow due to heat transfer is restricted to a laminar characteristic. To reveal changes in density variation, the same color scaling is used for the different pressure scenarios in figure 1. Moreover, the maximum and minimum values are directly given below the corresponding density field. The laminar fluid flow induced by convective heat transfer directly above the hot cylinder is clearly visible - independent of the pressure state. Furthermore, a reduction in pressure results in a decrease of $\Delta \rho=\rho_{\max }-\rho_{\min }$ for the specific pressure scenario. $\Delta \rho$ decreases from $\Delta \rho_{1013 \mathrm{mbar}}=1.18 \mathrm{~kg} \mathrm{~m}^{-3}-0.517 \mathrm{~kg} \mathrm{~m}^{-3}=0.663 \mathrm{~kg} \mathrm{~m}^{-3}$ for ambient pressure to $\Delta \rho_{355 \mathrm{mbar}}=0.233 \mathrm{~kg} \mathrm{~m}^{-3}$ for a pressure value of $355 \mathrm{mbar}$. If a linear density-refractive index coupling for air is hypothesized, ${ }^{2}$ the simulated decrease in $\Delta \rho$ would also lead to a decrease in the refractive index variation.

The simulation approach has been extended in a former conference contribution ${ }^{1}$ to quantify the effect of pressure reduction on light deflection, by coupling the density values to refractive index values by extrapolated Ciddor's equation ${ }^{3}$ for extreme temperatures above $100^{\circ} \mathrm{C}$. The obtained results confirmed a decrease in light deflection when lowering the surrounding pressure.

The main objective of this paper is the experimental validation of the previously presented simulation results in order to reveal the potential of a pressure reduction on the reachable accuracy of optical triangulation measurements of hot components. For this purpose, a low pressure measurement chamber has been developed at the 


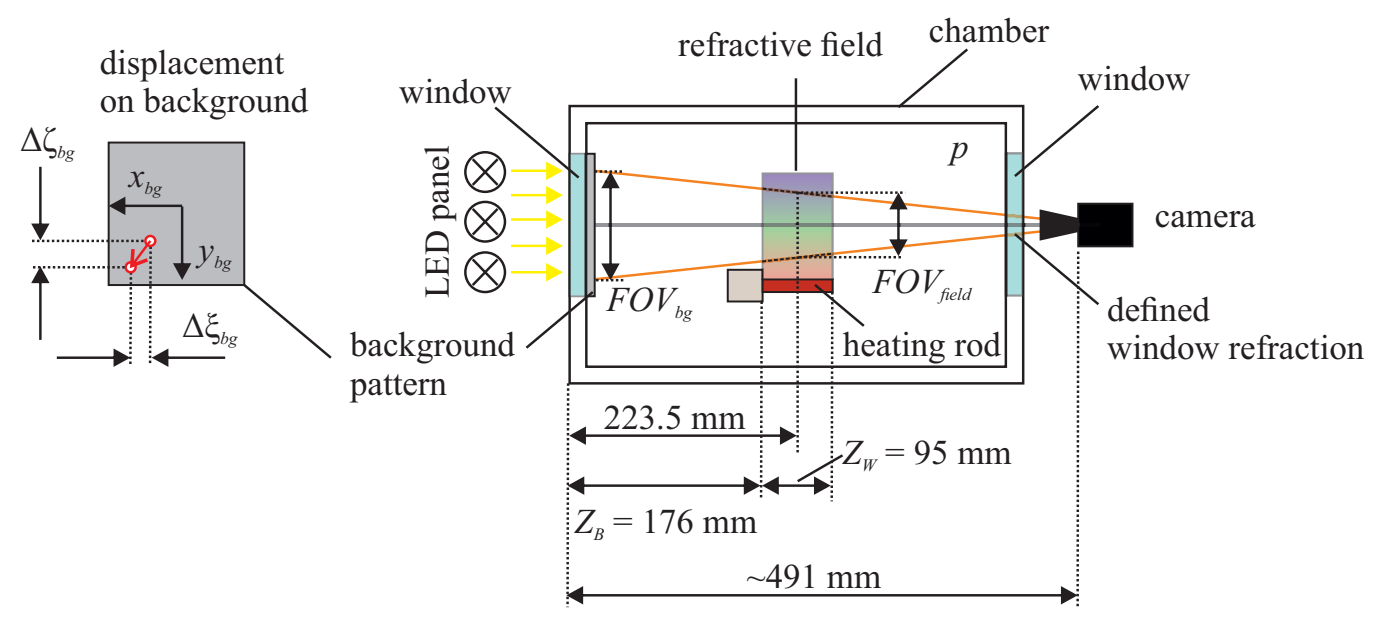

Figure 2: Principle outline of the background oriented schlieren (BOS) measurement setup in combination with a vacuum chamber. In order to reveal the effect of a pressure reduction on the inhomogeneous refractive index field around a hot object, a ceramic heating rod is place inside a vacuum chamber with pressure $p$. A background pattern is placed behind the inhomogeneous refractive field and is backlit by a LED panel. A camera acquires images of the refractive index field-affected background. The field of views (FOV) of the camera for the refractive field and the background are given as $F O V_{\text {field }} \approx 79 \mathrm{~mm} \cdot 79 \mathrm{~mm}$ and $F O V_{b g} \approx 145 \mathrm{~mm} \cdot 145 \mathrm{~mm}$. By comparing a reference image of the background (heating rod is switched off) with an affected background image (heating rod is switched on, a convective density flow manipulates the light paths), the 2D light ray displacement $\Delta \xi_{b g}$ and $\Delta \zeta_{b g}$ on the background is obtained by an optical flow algorithm. The displacement is evaluated for each camera pixel to gain a 2D refractive index field parallel to the camera sensor. To minimize light reflections due to the measurement windows, the background pattern is attached to the window from the chamber inside. The camera acquires images through a measurement window, as it cannot be placed inside the vacuum chamber. As it's effect on the light path is not only present in the reference image, but also in the images acquired under influence of the heating rod induced refraction, the window effect is not reproduced by an own refraction model.

Institute of Measurement and Automatic Control, allowing refractive index field measurements in different pressure states by the so-called background oriented schlieren (BOS) method. Experimental data of the 2D refractive index fields above a hot cylinder is presented in order to validate the hypothesized decrease in refractive index variations depending on the pressure state as suggested by the density simulation results according to figure 1.

\section{REFRACTIVE INDEX MEASUREMENT: THEORY AND PRINCIPLE OUTLINE OF BOS SETUP}

The BOS method permits quantitative 2D or - in more sophisticated setups - even 3D measurements of the refractive index of transparent media such as air. ${ }^{4,5}$ Light, traversing an optical inhomogeneous medium, will be refracted and bend towards optically more dense regions. If this refraction is indirectly captured by a camera in the form of a displacement value on a reference background, a refractive index value can be obtained by evaluating the associated displacement. Certain assumptions have to be made for the refractive index measurement and BOS setup used in this paper: The inhomogeneous field is only changing in a plane parallel to the camera sensor, but is not changing along the camera's optical axis. Furthermore, the paraxial approximation is supposed to be valid and the deflection angles in the refractive field are assumed to be small.

The principle outline of the developed BOS setup in combination with a vacuum chamber is depicted in figure 2 . The 2D BOS setup design is based on a former implementation, ${ }^{6}$ but is now adjusted to the specific low pressure application. It comprises a monochromatic camera and a heating rod that is used to generate an inhomogeneous refractive index field by convective density flow due to heat input into the surrounding air. A background pattern is placed behind the refractive field, in the camera sight cone. It is backlit by a LED panel, emitting white light to compensate for reduced camera light and to enhance the reachable contrast. By comparing a 
reference image of the background (heating rod at ambient temperature) with an affected background image (heating rod is switched on, a convective density flow manipulates the light paths), the light ray displacement on the background can be obtained by an appropriate algorithm - in this case an optical flow algorithm (Brox algorithm). ${ }^{7}$

To gain the associated refractive index value, the light ray displacement is weighted with a constant factor $k$ (see equation 1), that mainly depends on the geometric dimensions of the BOS setup. The relationship between light ray displacement on the background $\Delta \xi_{b g}$ in $x_{b g}$-direction and $\Delta \zeta_{b g}$ in $y_{b g}$-direction (compare to figure 2) and the refractive index gradients $\frac{\partial n}{\partial x}$ and $\frac{\partial n}{\partial y}$ in the inhomogeneous field are stated in equation 1 (according to Dalziel et al. ${ }^{8}$ ):

$$
\begin{aligned}
& {\left[\begin{array}{l}
\frac{\partial n}{\partial x} \\
\frac{\partial n}{\partial y}
\end{array}\right]_{\text {field }}=\underbrace{-\frac{2 n_{0}}{Z_{W}\left(Z_{W}+2 Z_{B}\right)}}_{\text {- }}\left[\begin{array}{c}
\Delta \xi \\
\Delta \zeta
\end{array}\right]_{b g},} \\
& {\left[\begin{array}{c}
\frac{\partial n}{\partial x} \\
\frac{\partial n}{\partial y}
\end{array}\right]_{\text {field }}=} \\
& k
\end{aligned}
$$

$Z_{B}$ parametrizes the distance between background and field and $Z_{W}$ defines the width of the field along the optical axis of the camera (compare to figure 2). $n_{0}$ is the refractive index of air without heat input and under ambient (chamber) conditions. As the geometrical parameters mainly define the weighting of the displacement values $\Delta \xi_{b g}$ and $\Delta \zeta_{b g}, n_{0}$ is set to 1 for all pressure scenarios.

Equation 1 can be transformed into Poisson's equation, which then can be numerically solved by applying appropriate boundary conditions. The result is a $2 \mathrm{D}$ scalar refractive index field, parallel to the camera sensor and gained from the measured vector gradient field according to equation 1.

The application of a vacuum chamber complicates the refractive index measurement, as the camera needs to acquire images through a measurement window (compare to figure 2). Light, traversing the glass window, will be deflected due to the discrete refractive index step, affecting the camera's light path. The main difference between the heat induced refraction in the inhomogeneous field and the discrete window refraction is the constancy of the window effect for a specific chamber pressure scenario. The window refraction is therefore defined and doesn't change over time. It is not corrected - for instance by an extended pinhole camera model considering window refraction. As it's effect on the camera's light path is present not only in the reference image, but as well in the images acquired under convective flow by the heating rod, this approach is considered to be legitimate.

Additional information on the basic BOS principles and the application of optical flow algorithms in BOS setups are stated in further literature. ${ }^{4,5,8-11}$

\section{EXPERIMENTAL SETUP}

The experimental setup is depicted in figure 3. It comprises an automated vacuum chamber with vacuum pump and measuring computer (figure 3,(1),(2),(3)), combined with a BOS measurement system to quantify the refractive index in different pressure states (figure 3,(4),(5a),(5b),(6)). The steel vacuum chamber (figure $3,(1)$ ) is equipped with a ventilation system, based on electro-pneumatic angle valves. A rotary valve vacuum pump enables a defined chamber vacuum production (figure 3,(2)). Two chamber windows permit optical measurements, and the illumination of the chamber inside. The BOS setup comprises a monochromatic camera with a resolution of 2048 pixel-2048 pixel, a 1"-CMOS-sensor and a $35 \mathrm{~mm}$ lens (figure 3,(4)). The inhomogeneous refractive index field is generated by a ceramic rod (figure 3,(6)), that can be heated up to temperatures of about $1020^{\circ} \mathrm{C}$ in $45 \mathrm{~s}$. The rod has a diameter of $11 \mathrm{~mm}$. The camera acquires images of a background with wavelet pattern (figure $3,(5 \mathrm{~b})$ ). It is illuminated through a chamber window by a LED panel (figure 3,(5a)). The pattern is printed on an adhesive foil, and directly fixed to the right window inside the chamber. This approach avoids window light reflections, that might occur when placing the pattern behind the window outside the chamber. A measuring computer allows an automated operation of vacuum production and the management of the BOS system (figure 3,(3)).

The geometric distances of the BOS setup are very important for the transformation of the pixel displacement into the refractive index gradient field. The distance values are given in figure 2 with $Z_{W}=95 \mathrm{~mm}$ and $Z_{B}=$ $176 \mathrm{~mm}$. The resulting $k$-factor is $-4.71 \times 10^{-5} \mathrm{~mm}^{-2}$, if $n_{0}$ is approximated with a value of 1 . The detectable displacement field is defined by the camera sight cone in the middle of the refractive field $\left(F O V_{\text {field }} \approx 79 \mathrm{~mm}\right.$. 


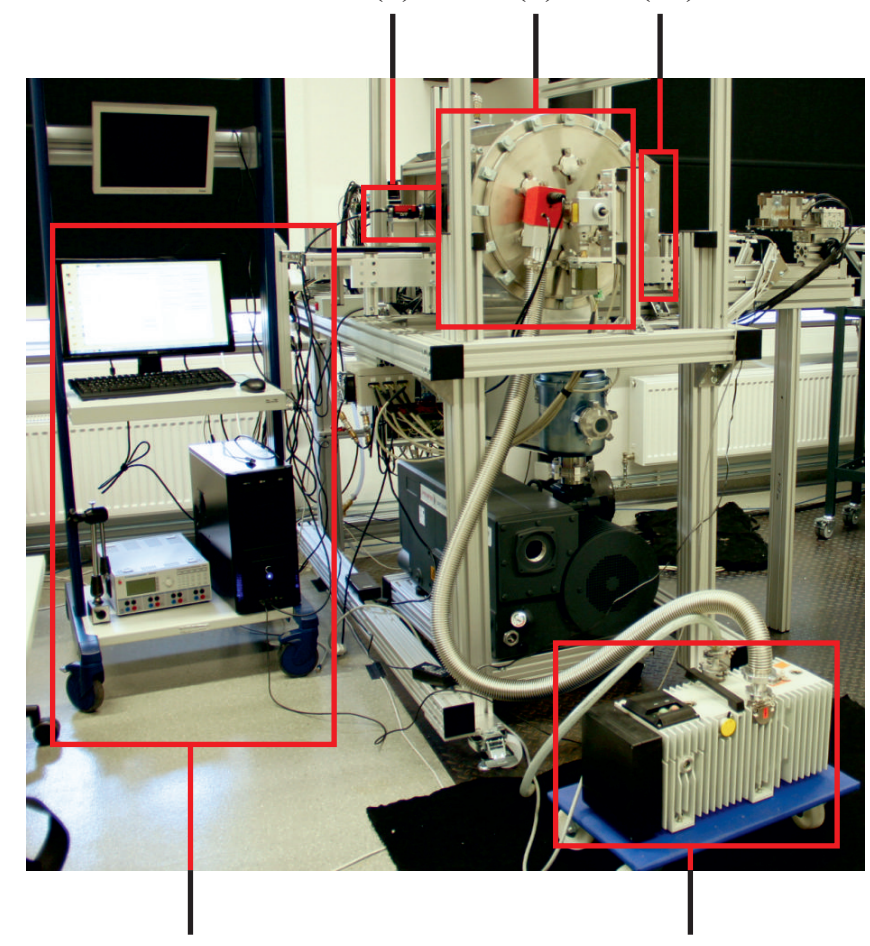

(3)

(2)

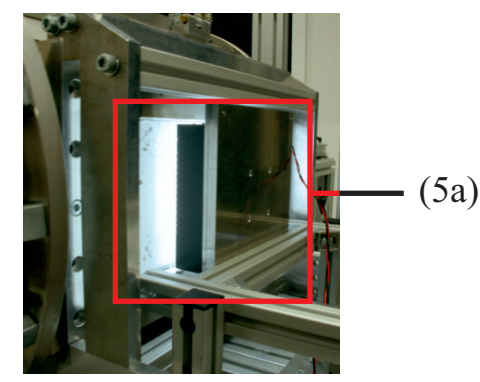

(5b)

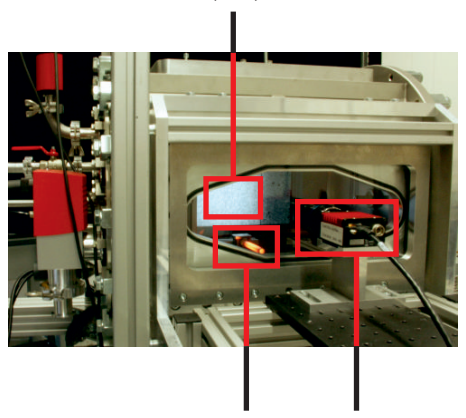

(6)

(4)

Figure 3: Experimental setup comprising a vacuum chamber with measurement windows and electro-pneumatic angle valves for ventilation (1), a rotary valve vacuum pump (2), a measuring computer (3) for the management of the test stand (e.g. the ventilation system) and the BOS measurements, a monochromatic measurement camera with $35 \mathrm{~mm}$ lens (4) and a background-pattern (5b) backlit by a LED panel (5a). The inhomogeneous refractive index field is built up by a ceramic heating rod (6), that can be heated up to temperatures of approximately $1020^{\circ} \mathrm{C}$ within $45 \mathrm{~s}$.

$79 \mathrm{~mm}$, compare to figure 2). The detectable field area can be gained by simple plane-to-plane transformations from camera sensor plane to field plane. The measurable refractive index field is smaller, as the displacement values for boundary pixels are excluded from the refractive index calculation.

\section{MEASUREMENT EXECUTION}

The refractive index field above the heating rod is measured at four different chamber pressure states: at ambient pressure $\left(p_{1}=987 \mathrm{mbar}\right)$, and at three reduced pressure states $\left(p_{2} \approx 510 \mathrm{mbar}, p_{3} \approx 340 \mathrm{mbar}, p_{4} \approx 63 \mathrm{mbar}\right)$. As the measurement windows rest on O-ring seals, a translational movement of the windows due to pressure reduction cannot be excluded. Therefore, a reference image is taken for each specific pressure scenario before the heating rod is switched on. The rod develops it's full heat after approximately $45 \mathrm{~s}$. The heat-up process is observed with the camera by acquiring at least 150 images with a frame rate of $1 \mathrm{~Hz}$ after turning the rod on. Hereby, it is guaranteed, that the maximum expansion and magnitude of the refractive index field is documented. The reduced pressure states only correspond to a pressure range, as the pressure values slightly change after the heating rod is switched on. This is due to the expansion of heated up air and cannot be avoided.

To identify the measurement image, that possibly results in the maximum refractive index variation, the respective image with the maximum 2D norm displacement value $d=\sqrt{\Delta \xi^{2}+\Delta \zeta^{2}}$ in a measurement series is chosen. This selection procedure is meant to guarantee the comparability of the different refractive index fields at different pressure states. A simple time-wise selection is not representative, as the switching on of the heating rod is realized manually and not directly coupled to the starting of the BOS measurement. 
legend: displacement field

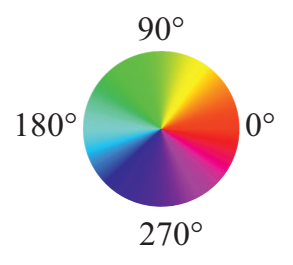

HSV color space: max. saturation for displacement magnitude of 10 pixel
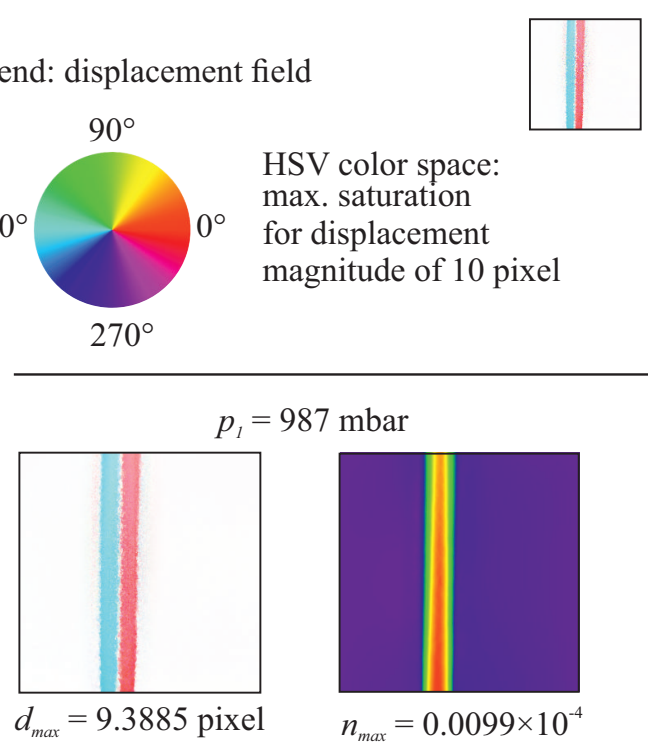

$p_{1}=987 \mathrm{mbar}$

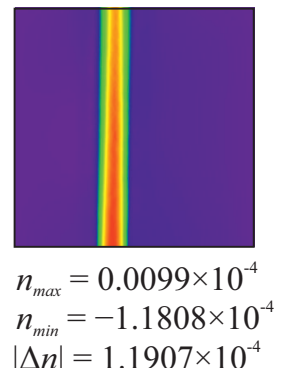

$p_{2}=502 \ldots 518 \mathrm{mbar}$

legend: refractive index field

$n_{\text {max }}=0.0423 \times 10^{-4}$

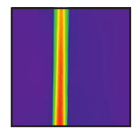

$n_{\text {min }}=-1.1808 \times 10^{-4}$
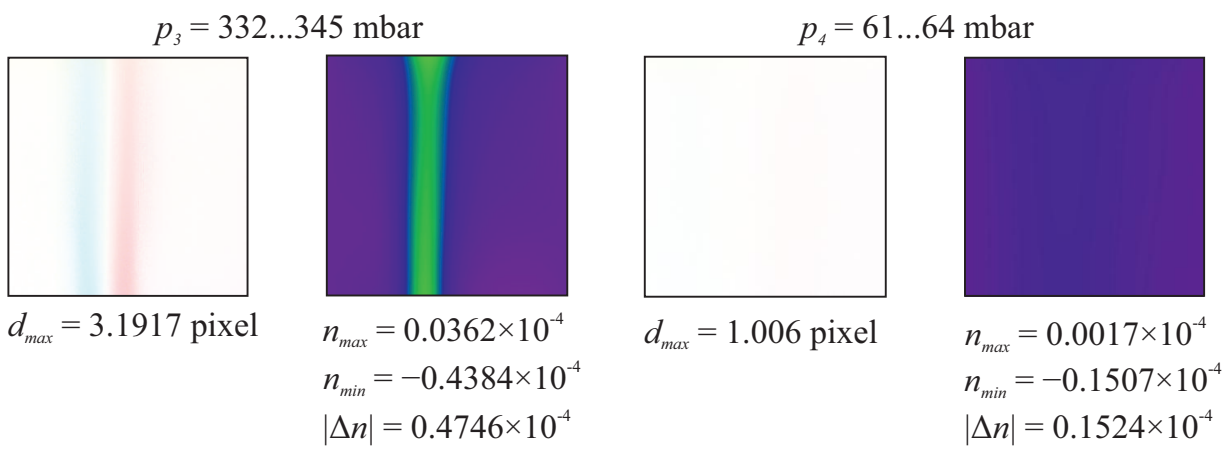

Figure 4: Measurement results for the displacement maps on the camera sensor in pixel and the corresponding 2D scalar refractive index fields for different pressure states. The displacement is of vector representation and is therefore given in the HSV color space, encoding the displacement direction with a specific color, and the magnitude $d=\sqrt{\Delta \xi^{2}+\Delta \zeta^{2}}$ by color saturation. The maximum displacement value $d_{\max }$ is directly given under the corresponding graph. The refractive index fields have been derived based on a Dirichlet boundary condition for the left and right sides with $n_{0}=0$ for all pressure states. The maximum and minimum values are also given directly under the corresponding field, next to the maximum variation $|\Delta n|$.

\section{RESULTS AND DISCUSSION}

The measurement results are given in figure 4, with the legends at the top. The displacement field legend is depicted on the left side, the legend for the refractive index field on the right side. The results for a specific pressure value (from $p_{1}=987$ mbar to $p_{4} \approx 63$ mbar) are presented in pairs: next to the displacement field (on the camera sensor in pixel), the corresponding refractive index field is cited. As the displacement is of vector representation, it is given in the HSV color space. The orientation of a displacement vector is given in a specific color (blue: displacement to the left, red: displacement to the right, compare to displacement field legend in figure 4). The 2D norm of the displacement vector is encoded by the color saturation. The displacement maps are scaled to the same maximum value of 10 pixel to allow a direct comparison between the different pressure measurements. 
The refractive index fields are of scalar representation and are derived form the refractive gradient fields by integration, which in turn are derived from the displacement maps. Appropriate boundary conditions are necessary when integrating. In this case the so-called Dirichlet condition is applied to the left and right side of the evaluated refractive index area, meaning a fixed refractive index value $n_{0}$ has to be chosen. As the $n_{0}$-value for the different pressure values are not known, the Dirichlet condition is set to 0 for all pressure scenarios. In this case, the resulting refractive index field is not given in absolute terms, but only illustrates the variation of the refractive index $\Delta n$. This is legitimate, as the validation of the simulations in figure 1 can be considered successful, if a decrease in $\Delta n$ can be confirmed when lowering the pressure. There is no need to absolutely quantify the refractive index. The upper and lower boundary conditions are defined by the so-called Neumann condition. Further information on the discrete integration of the vector gradient field and the applied boundary conditions (e.g. the Neumann condition for the upper and lower boundaries) to derive the scalar refractive index field are given in previous work. ${ }^{6}$

The displacement maps in figure 4 indicate the effect of the pressure reduction: A reduction leads to a decrease of light refraction in the inhomogeneous refractive index field of the heating rod and therefore to smaller displacement values.

The maximum pixel displacement $d_{\max }=\sqrt{\Delta \xi_{\max }{ }^{2}+\Delta \zeta_{\max }{ }^{2}}$ occurs at ambient pressure $\left(p_{1}=987 \mathrm{mbar}\right.$, $d_{\max }=9.3885$ pixel $)$, whereas the lowest maximum value occurs for the lowest pressure value $\left(p_{4}=61 \ldots 64\right.$ mbar, $d_{\max }=1.006$ pixel). Moreover, the derived refractive index fields show the same characteristic for all pressure scenarios: A laminar and more or less symmetric flow can be observed above the heating rod. This result corresponds to the density simulations in figure 1: Although the simulations have been derived for a different steel cylinder diameter and temperature, the qualitative shape of the convective density flow above the heating rod is the same.

The variation of the refractive index $\Delta n$ is maximal for ambient pressure $|\Delta n|=1.1907 \times 10^{-4}$, mainly caused by the magnitude $\left|n_{\min }\right|$ of $1.1808 \times 10^{-4}$. $\left|n_{\min }\right|$ decreases to a value of $\left|n_{\min }\right|=0.1507 \times 10^{-4}$ in case of the lowest chamber pressure $p_{4}$.

$n_{\max }$ varies for all pressure scenarios without identifiable trend. An increase of the refractive index hasn't been expected by the authors, as a heat input normally results in a density reduction in air, and therefore in a reduction of the refractive index. As the magnitude $\left|n_{\max }\right|$ is at least 10 times smaller than the magnitude of $\left|n_{\min }\right|$ (for all pressure scenarios), it's effect is considered to be negligible compared to the logical consistency of the $\left|n_{\text {min }}\right|$ values. The reason for $n$ values greater than 0 is possibly the violation of the BOS assumption, that the refractive index field is not changing along the camera's optical axis. If the convective density flow above the heating rod is slightly flaring, the displacement measurements are disturbed, which in turn might result in disturbed refractive index results.

\section{CONCLUSION AND FUTURE WORK}

The presented light displacement and refractive index field measurements at different pressure scenarios confirm the simulation results presented in figure 1: A pressure reduction results in a homogenization of the refractive index field around a hot object, as the refractive index variation in terms of $\Delta n$ is decreased. The developed vacuum chamber combined with the presented BOS system allows the documentation of the hypothesized effect. An improvement of the developed measurement setup could be achieved by an implementation of an appropriate camera model, respecting the discrete window refraction.

If the geometry of wrought hot components is meant to be measured with high precision, optical inhomogeneity must be avoided. The developed low pressure chamber allows a homogenization of the heat induced refractive index field around hot measurement objects, and therefore restores a critical requirement for optical triangulation measurements: a defined light propagation. The suggested approach enables the quantification of material distortion effects during air-cooling, which is of special interest when measuring hybrid materials with differing thermal expansion coefficients. In this specific application particularly the component's shrinkage behavior can help to classify geometry deformation after forming. One basic requirement for this kind of shrinkage measurements is the rapid feeding of the hot measurement object into the low pressure chamber, a rapid 
chamber evacuation and the usage of an optical measurement system that accounts for the defined and discrete light refraction induced by the necessary chamber window. An appropriate fringe projection system for this task is currently being developed at the Institute of Measurement and Automatic Control.

\section{ACKNOWLEDGMENTS}

The results presented in this paper were obtained within the Collaborative Research Centre 1153 Process Chain for Manufacturing Hybrid High Performance Components by Tailored Forming in the subproject C5 Multiscale Geometry Inspection of Joining Zones. The authors would like to thank the German Research Foundation (DFG) for the financial and organisational support of this project.

\section{REFERENCES}

[1] Beermann, R., Quentin, L., Kästner, M., Pösch, A., and Reithmeier, E., "Simulation of light deflection due to an inhomogeneous refractive index field induced by a hot measurement object," in [DGaO-Proceedings 2017], DGaO (2017).

[2] Dale, T. P. and Gladstone, J., "On the influence of temperature on the refraction of light," Phil. Trans. R. Soc. Lond. 148, 887-894 (1858).

[3] Ciddor, P. E., "Refractive index of air: new equations for the visible and near infrared," Appl. Opt. 35, 1566-1573 (Mar 1996).

[4] Meier, G., "Hintergrund-Schlierenverfahren. Deutsche Patentanmeldung DE 19942856A1," (1999).

[5] Goldhahn, E., Alhaj, O., Herbst, F., and Seume, J., "Quantitative measurements of three-dimensional density fields using the background oriented schlieren technique," in [Imaging Measurement Methods for Flow Analysis], Nitsche, W. and Dobriloff, C., eds., 135-144, Springer Berlin Heidelberg, Berlin, Heidelberg (2009).

[6] Beermann, R., Quentin, L., Pösch, A., Reithmeier, E., and Kästner, M., "Background oriented schlieren measurement of the refractive index field of air induced by a hot, cylindrical measurement object," Appl. Opt. 56, 4168-4179 (May 2017).

[7] Brox, T., Bruhn, A., Papenberg, N., and Weickert, J., "High accuracy optical flow estimation based on a theory for warping," in [European conference on computer vision], 25-36, Springer (2004).

[8] Dalziel, S. B., Hughes, G. O., and Sutherland, B. R., "Whole-field density measurements by synthetic schlieren," Experiments in Fluids 28(4), 322-335 (2000).

[9] Venkatakrishnan, L. and Meier, G., "Density measurements using the background oriented schlieren technique," Experiments in Fluids 37, 237-247 (2004).

[10] Raffel, M., "Background-oriented schlieren (bos) techniques," Experiments in Fluids 56, 60 (Mar 2015).

[11] Atcheson, B. and Heidrich, W., "An evaluation of optical flow algorithms for background oriented schlieren imaging," Experiments in Fluids 46, 467-476 (2009). 